\title{
ESTRATÉGIAS DE COPING EM ATLETAS DE FUTEBOL FEMININO: ESTUDO COMPARATIVO
}

COPING STRATEGIES IN WOMEN'S SOCCER ATHLETES: A COMPARATIVE STUDY

ESTRATEGIAS DE COPING EN ATLETAS DE FÚTBOL FEMENINO: UN ESTUDIO COMPARATIVO

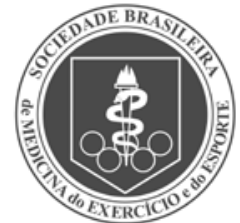

Artigo Original

Original ARtICle Artículo Original
Matheus Rizzato Rossi (Acadêmico do curso de Medicina)

Luciano Magalhães Vitorino² (Enfermeiro)

Ricardo Pombo Salles ${ }^{3}$

(Educador Físico)

Paulo José Oliveira Cortez ${ }^{1}$

(Fisioterapeuta)

1. Faculdade de Medicina de Itajubá, Itajubá, MG, Brasil.

2. Universidade Federal de São Paulo, Escola Paulista de Enfermagem, Itajubá, MG, Brasil. 3. Universidade Trás-os-Montes e Alto Douro, Vila Real, Portugal.

\section{Correspondência:}

Faculdade de Medicina de Itajubá Avenida Renó Júnior, 368, Bairro São Vicente, Itajubá, MG, Brasil. 37502-138. matheus_rrossi@hotmail.com

\section{RESUMO}

Introdução: Os atletas de alto rendimento sofrem com uma série de fatores causadores de perturbações psicológicas, que podem acarretar danos ao seu desempenho final. Com a competitividade elevada e o nivelamento nos treinamentos físico e tático, as estratégias de coping (enfrentamento) para superar essas perturbações podem fazer a diferença entre um elenco campeão ou perdedor. Objetivos: Analisar e comparar as estratégias de coping entre atletas de alto rendimento e praticantes de futebol feminino. Métodos: Trata-se de um estudo quantitativo, descritivo, transversal e com amostragem não probabilística. A amostra foi composta por 56 atletas, divididas em dois grupos: G1 - atletas de alto rendimento e G2 - praticantes de futebol feminino. O instrumento utilizado foi o Athletic Coping Skills Inventory-28 (ACSI-28), validado para o Brasil (ACSI-25BR) e um questionário sociodemográfico contendo 12 questões, elaborado pelos próprios autores. Para a análise dos dados foi usada a estatística descritiva, teste de normalidade de Shapiro-Wilk e o teste $t$ de Student para dados independentes. O nível de confiança adotado foi de 95\%. Resultados: Atletas de alto rendimento obtiveram maior pontuação média, estatisticamente significante, com relação às praticantes de futebol feminino nas dimensões: "desempenho sob pressão" ( $p=0,048)$, "concentração" $(p=0,020)$ e "confiança/motivação" ( $(p=0,009)$. Conclusão: Atletas de alto rendimento obtiveram melhor desempenho em todas as dimensões, exceto em "treinabilidade" e "ausência de preocupação", quando comparadas ao grupo de praticantes de futebol feminino.

Descritores: atletas; futebol; adaptação psicológica.

\section{ABSTRACT}

Introduction: High-performance athletes suffer a series of psychological disorders that can harm their overall performance. With the high levels of competitiveness and physical/tactical training that are required today, coping strategies to overcome these psychological disorders can make the difference between a winning team and a losing team. Objective: To compare coping strategies among high-performance athletes and amateur women's soccer players. Methods: This is a quantitative, descriptive, cross-sectional, non-probability study. The sample consisted of 56 athletes, divided into two groups: G1 - high-performance athletes and G2 - amateur women's soccer players. The instrument used was the Athletic Coping Skills Inventory-28 (ACSI - 28), validated for Brazil (ACSI - 25BR) and a demographic questionnaire containing 12 questions, developed by the authors themselves. For the data analysis, descriptive statistics, the Shapiro- Wilk test and the Student $t$ test for independent data were used. A confidence level of $95 \%$ was adopted. Results: The high performance athletes had higher average scores, which were statistically significant, comparing to the amateur athletes, in the dimensions: "performance under pressure" ( $p=0.048)$, "concentration" $(p=0.020)$ and "confidence/motivation" $(p=0.009)$. Conclusion: The high performance athletes performed better in all dimensions except for "trainability" and "freedom from worry", when compared to the amateur athletes.

Keywords: athletes; soccer; adaptation, psychological.

\section{RESUMEN}

Introducción: Las atletas de alto rendimiento padecen de una serie de factores que causan trastornos psicológicos que pueden causar daños a su rendimiento final. Con la alta competitividad y nivelación en el entrenamiento físico y táctico, las estrategias de coping (afrontamiento) para superar estos trastornos pueden hacer la diferencia entre un equipo campeón o un perdedor. Objetivos: Analizar y comparar las estrategias de coping (afrontamiento) entre las atletas de alto rendimiento y aficionadas de fútbol femenino. Métodos: Se trata de un muestreo cuantitativo, descriptivo, transversal y no probabilístico. La muestra estaba compuesta por 56 atletas, divididas en dos grupos: G1 - atletas profesionales y G2 - jugadoras aficionadas de fútbol femenino. El instrumento utilizado fue el Athletic Coping Skills Inventory-28 (ACSI-28), validado para Brasil (ACSI-25BR) y un cuestionario sociodemográfico que contenía 12 preguntas preparadas por los autores. Para el análisis de datos se utilizó la estadística descriptiva, prueba de normalidad de Shapiro-Wilk y la prueba t de Student para datos 
independientes. El nivel de confianza adoptado fue 95\%. Resultados: Las atletas de alto rendimiento tuvieron puntuaciones medias más elevadas, estadísticamente significativas en relación con las jugadoras de fútbol femenino en las dimensiones: "rendimiento bajo presión" ( $p=0,048)$, "concentración" $(p=0,020)$ y "confianzal motivación" $(p=0,009)$. Conclusión: Las atletas de alto rendimiento obtuvieron mejores resultados en todas las dimensiones excepto en "capacidad de entrenamiento" y "ausencia de preocupación", en comparación con el grupo de jugadoras de fútbol femenino.

Descriptores: atletas; fútbol; adaptación psicológica.

\section{INTRODUÇÃO}

Atualmente, a necessidade de se extrair o máximo de desempenho do atleta durante a prática esportiva de alto rendimento, despertou o interesse de profissionais como psicólogos, médicos e especialistas do desporto em relação à influência do estresse e à maneira com que cada um lida com ele $\mathrm{e}^{1-5}$.

Os diferentes tipos de pressão que o atleta de alto desempenho sofre, não só no ambiente de competição, oriundos de treinadores e torcedores, mas também no ambiente familiar, podem fazer com que este, se não bem preparado psicologicamente, desenvolva elevado nível de estresse. Isto pode se tornar um redutor do desempenho esportivo, uma vez que está associado ao aumento da tensão muscular e à criação de déficits de atenção ${ }^{6}$.

De acordo com Lazarus e Folkman7, uma situação é considerada mais ou menos estressante a partir de uma auto avaliação que a pessoa realiza, e o significado final que esta representa. Portanto, como os indivíduos são diferentes, haverá interpretações diferentes, ou seja, o que afeta uma pessoa pode ser indiferente para outra ${ }^{8}$.

Um evento é considerado estressante quando causador de prejuízo ou dano, e neste instante os sujeitos buscam estratégias para se adaptarem ao momento ${ }^{8}$. Segundo Sordes-Arder et al. ${ }^{9}$, para superar este conflito gerado pela situação estressante o indivíduo emprega estratégias de coping. Tais estratégias são descritas como o conjunto de ações utilizadas pelas pessoas para adaptarem-se a circunstâncias adversas ou estressantes ${ }^{10}$.

De acordo com Ryan-Wenger ${ }^{11}$, estratégias de enfrentamento são ações conscientes, que podem ser aprendidas, usadas e descartadas e cujo objetivo é lidar com o estresse percebido. Constituem-se, portanto, em um processo flexível e intencional, orientado para o futuro, na busca do alívio do estresse. Assim, acredita-se que os atletas de sucesso serão aqueles que possuem estratégias mais eficazes de enfrentamento durante situações estressantes, que são inerentes à prática esportiva ${ }^{12,13}$.

No Brasil, segundo a Confederação Brasileira de Futebol (CBF), existem cerca de 400 mil mulheres jogando futebol ${ }^{14}$. Embora desprestigiado pela grande mídia e sem os grandes investimentos como os observados no futebol masculino, a modalidade vem crescendo a passos lentos, com importantes resultados, tanto em termos de clubes, quanto em termos de seleção (Copa do Mundo de Futebol Feminino, Jogos Pan-Americanos e Olimpíadas), sendo sempre esperança de medalhas.

Diante desse contexto, fica evidente a relevância de se verificar o nível desta habilidade psicológica, visando seu aprimoramento, como uma maneira de melhorar o desempenho nacional em competições de alto rendimento. Neste sentido, o objetivo foi analisar e comparar as estratégias de coping de atletas de futebol feminino que se enquadram na prática do esporte de alto rendimento e de atletas que não possuem este nível competitivo (praticantes).

\section{MÉTODOS}

Realizou-se um estudo quantitativo, transversal e com amostragem não probabilística. A amostra total foi constituída por 56 atletas, dispostas em dois grupos: Grupo 1 (G1)- Refere a atletas pertencentes ao elenco de futebol feminino do São José Esporte Clube $(n=28)$. Grupo 2 (G2) - praticantes de futebol feminino, da cidade de São José dos Campos - SP, que não se enquadram no esporte de alto rendimento $(n=28)$. A coleta de dados ocorreu entre setembro de 2014 e janeiro de 2015.

O projeto de pesquisa obedeceu aos preceitos éticos da Resolução do Conselho Nacional de Saúde 466/2012, sendo aprovado pelo Comitê de Ética da Faculdade de Medicina de Itajubá, Minas Gerais. Responsáveis pelo clube e as atletas avaliadas assinaram o Termo de Consentimento Livre e Esclarecido (TCLE), comprovando a anuência em participar do estudo.

Os instrumentos para coleta de dados foram: a) questionário para caracterização sociodemográfica, construído pelos próprios autores, composto por 12 questões fechadas, contendo informações como idade, escolaridade, hábitos, tempo de prática e situação financeira. b) para as estratégias de coping foi utilizado o instrumento versão brasileira do Athletic Coping Skills Inventory-28 (ACSI-25BR) ${ }^{15}$, desenvolvido originalmente por Smith et al. ${ }^{16}$. Trata-se de um questionário composto por 28 itens, que englobam pensamentos e ações as quais as pessoas utilizam para lidar com demandas internas ou externas no contexto esportivo. As respostas se dão em uma escala Likert de quatro pontos, variando de zero ("quase nunca") a três ("quase sempre"), apresentando sete subescalas, o qual avalia os seguintes aspectos psicológicos: 1) lidar com adversidades; 2) desempenho sob pressão; 3) metas/preparação mental; 4) concentração; 5) ausência de preocupação; 6) confiança/Motivação; 7) treinabilidade. As pontuações mais altas significam valores mais elevados de coping em cada umas das dimensões. Na validação brasileira o alfa de Cronbach do instrumento variou de 0,72 a 0,79, evidenciando boa consistência interna dos dados. Na versão brasileira, três itens foram removidos da análise por não apresentarem cargas fatoriais suficientes para pontuarem em alguma das dimensões ${ }^{17}$.

Como critério de inclusão, para o grupo das atletas de alto rendimento, era necessária idade superior a 18 anos e vínculo com o São José Esporte Clube há pelo menos três meses, uma vez que há evidências de que os tipos estratégias de coping empregados pelo individuo surgem a partir de três meses após o contato com o fator estressante ${ }^{18}$. Já para as praticantes de futebol feminino, era necessário idade também superior a 18 anos e praticar a modalidade com frequência mínima de uma vez na semana, por pelo menos três meses, na cidade de São José dos Campos (SP).

Para a análise estatística, as variáveis referentes ao perfil das atletas foram descritas em frequência absoluta e relativa. A verificação da normalidade foi realizada através do uso do teste de Shapiro-Wilk. 
Na análise das diferenças entre os desfechos de coping entre os dois grupos, utilizou-se teste $t$ de Student para dados independentes. 0 nível de confiança adotado foi de 95\% ( $p<0,05)$.

\section{RESULTADOS}

Os resultados apresentados na Tabela 1 evidenciam que em ambos os grupos a idade predominante está na faixa de 18 a 20 anos; os níveis de escolaridade também se mostram equiparados, destacando-se que cerca de $50 \%$ das atletas de alto rendimento ingressaram no ensino superior. Todas as atletas pertencentes ao elenco do São José Esporte Clube se dedicam integralmente à prática esportiva, sem possuir outra fonte de renda, com importante taxa de insatisfação com a remuneração (75\%).

Com relação à prática esportiva, 46,5\% das atletas de alto rendimento está há mais de 24 meses no clube, sendo que todas possuem treinos quatro vezes na semana e disputam campeonatos de âmbito nacional. Diferentemente das praticantes, em que o tempo de permanência no clube atual é menor, a frequência de treinos é em torno de duas a três vezes na semana e participam de torneios de nível municipal/regional (Tabela 2).

Tabela 1. Idade, escolaridade, remuneração, outras atividades empregatícias e satisfação das atletas com renda.

\begin{tabular}{|c|c|c|}
\hline Características & $\begin{array}{l}\text { Alto rendimento (G1) } \\
\qquad(n=28)\end{array}$ & $\begin{array}{l}\text { Praticantes (G2) } \\
\qquad(n=28)\end{array}$ \\
\hline Idade (anos) & $\mathrm{N} \%$ & $\mathrm{~N} \%$ \\
\hline Entre 18 e 20 & $12(42,9)$ & $16(57,2)$ \\
\hline Entre 21 e 25 & $6(21,4)$ & $9(32,1)$ \\
\hline Entre 26 e 30 & $7(25,0)$ & $2(7,1)$ \\
\hline Maior que 30 & $3(10,7)$ & $1(3,6)$ \\
\hline \multicolumn{3}{|l|}{ Escolaridade } \\
\hline Ensino fundamental incompleto & $1(3,6)$ & $0(0,0)$ \\
\hline Ensino fundamental completo & $1(3,6)$ & $0(0,0)$ \\
\hline Ensino médio incompleto & $4(14,3)$ & $1(3,6)$ \\
\hline Ensino médio completo & $8(28,5)$ & $19(67,9)$ \\
\hline Ensino superior incompleto & $7(25,0)$ & $5(17,8)$ \\
\hline Ensino superior completo & $7(25,0)$ & $3(10,7)$ \\
\hline \multicolumn{3}{|l|}{ Remuneração pelo esporte } \\
\hline Sim & $28(100,0)$ & $0(0,0)$ \\
\hline Não & $0(0,0)$ & $28(100,0)$ \\
\hline \multicolumn{3}{|l|}{ Outro emprego } \\
\hline $\operatorname{Sim}$ & $0(0,0)$ & $15(53,6)$ \\
\hline Não & $28(100,0)$ & $13(46,4)$ \\
\hline \multicolumn{3}{|l|}{ Renda suficiente } \\
\hline $\operatorname{Sim}$ & $7(25,0)$ & $16(57,1)$ \\
\hline Não & $21(75,0)$ & $12(42,9)$ \\
\hline
\end{tabular}


Atletas de alto rendimento obtiveram maior pontuação média em relação às praticantes de futebol feminino nas dimensões: desempenho sob pressão $(p=0,048)$, concentração $(p=0,020)$ e confiança/motivação ( $p=0,009 ;$ Tabela 4).

Tabela 4. Estratégias de coping nas atletas de alto rendimento e nas praticantes de Futebol Feminino.

\begin{tabular}{c|c|c|c}
\hline Estratégias & $\begin{array}{c}\text { Alto rendimento } \\
\text { (média IC95\%) }\end{array}$ & $\begin{array}{c}\text { Praticantes } \\
\text { (média IC95\%) }\end{array}$ & $\mathbf{p}^{*}$ \\
\hline Lidar com Adversidades & $6,9(6,0-7,9)$ & $6,4(5,7-7,1)$ & 0,320 \\
\hline Desempenho sob Pressão & $7,2(6,1-8,3)$ & $6,1(5,6-6,5)$ & 0,048 \\
\hline Metas/Preparação Mental & $8,0(7,1-8,9)$ & $7,3(6,3-8,3)$ & 0,273 \\
\hline Concentração & $6,2(5,5-6,9)$ & $5,1(4,6-5,7)$ & 0,020 \\
\hline Ausência de Preocupação & $8,3(7,2-9,4)$ & $8,8(7,9-9,6)$ & 0,461 \\
\hline Confiança/Motivação & $7,1(6,5-7,7)$ & $6,0(5,4-6,6)$ & 0,009 \\
\hline Treinabilidade & $7,5(6,7-8,3)$ & $8,4(7,7-9,1)$ & 0,075 \\
\hline
\end{tabular}

* Valor de probabilidade referente ao teste t de Student para dados independentes.

\section{DISCUSSÃO}

A proposta deste estudo foi analisar e comparar as estratégias de coping através do ACSI-25BR em atletas de futebol feminino de alto rendimento e em praticantes do esporte. Em relação às dimensões do instrumento, observou-se que as atletas pertencentes ao alto rendimento apresentaram maior uso das estratégias de coping em: desempenho sob pressão, concentração e confiança/motivação.

Altos valores na dimensão desempenho sob pressão significa que estes atletas, ao serem expostos a situações extremas, se sentem desafiados, estimulados, obtendo melhor rendimento nestas ocasiões ${ }^{15,19}$. Essa característica psicológica é fundamental para atletas de alto rendimento que por diversas vezes se encontram em momentos decisivos, em que um erro pode determinar a derrota.

Aliada a esta habilidade, a dimensão confiança/motivação, está relacionada à capacidade do atleta de acreditar em seu potencial, permanecendo positivo e motivado com seu desempenho, mesmo em situações adversas. Já a concentração se associa ao foco do atleta, não se distraindo facilmente, por ocasiões inesperadas ou pressões externas 15,19,20.

Assim, estas atletas de alto rendimento, ao se depararem com situações adversas, se sentem estimuladas a atingir melhores níveis de desempenho, elevando o nível de concentração e sempre acreditando em seu potencial e no de suas companheiras.

Os resultados apresentados corroboram com o estudo de Coimbra et al. ${ }^{21}$ que utilizou uma amostra com 375 atletas, em que independente do gênero e modalidade esportiva, atletas com melhores níveis competitivos utilizam-se melhor das dimensões, lidar com adversidades, metas/preparação mental, livre de preocupações e confiança/motivação.

É importante salientar que nas dimensões ausência de preocupações e treinabilidade, apesar de não estatisticamente significante, as praticantes de futebol feminino obtiveram melhores pontuações médias. A dimensão treinabilidade avalia se as atletas estão dispostas a aprender com as instruções dos treinadores, se aceitam criticas como construtivas, sem tomá-las como pessoais e sem se aborrecer ${ }^{15}$.

Atletas de alto rendimento tendem a obter menores pontuações nesta dimensão ao serem comparados com aqueles de níveis inferiores ${ }^{21}$. Tal afirmação é corroborada com o estudo de Nascimento 22 , que, ao verificar as estratégias de coping em atletas da categoria juvenil e infantil de futebol de campo, percebeu que as estratégias mais utilizadas por ambas as categorias eram: treinabilidade e confiança. Porém, um estudo comparando atletas convocados e não convocados para a seleção brasileira de futsal masculino, observou que desempenho sob pressão, confiança/motivação e treinabilidade são mais utilizados pelos jogadores convocados ${ }^{23}$.

Ausência de preocupações pode ser definida como a habilidade de não se colocar preocupação desnecessária em seu desempenho esportivo ${ }^{15}$. O principal fator que pode ter resultado em uma pontuação relativamente baixa para as atletas de alto rendimento do futebol feminino - (que) diferentemente do futebol masculino - vivem à margem dos grandes montantes de dinheiro e possuem como fonte exclusiva de renda o esporte, são as questões financeiras. Dessa maneira, estes problemas extracampo podem gerar uma autocobrança excessiva, para que desempenhem bem sua função e se transfiram para um país em que esta modalidade seja mais reconhecida.

De acordo com alguns autores ${ }^{24}$, a capacidade de utilizar estratégias de coping influencia de modo proporcional na assimilação de situações possivelmente estressantes. É necessário dizer que, quanto maior o número de estratégias que o sujeito desenvolve, menor é a sensação de instabilidade perante as estas situações ${ }^{22}$.

Para Coimbra et al. ${ }^{21}$, a grande diferença entre atletas de diferentes níveis competitivos são a quantidade e a qualidade das competições. Isso sugere que a experiência dos esportistas é fundamental, pois a partir daí passam a utilizar estratégias mais adequadas e efetivas para enfrentamento dos problemas ${ }^{25}$. Dessa maneira, esse pensamento segue o raciocínio de Ryan-Wenger ${ }^{11}$, em que o coping é aperfeiçoado através de um processo de aprendizagem constante e quanto mais preparado o atleta, melhor será o seu desempenho diante de uma situação perturbadora.

Diversas pesquisas apontam para esta linha de raciocínio, como a de Nicholls et al. ${ }^{26}$, o qual concluiu que atletas de alto rendimento, independente da modalidade, são mais eficientes ao utilizar as estratégias de coping. Mahoney et al. ${ }^{27}$, numa amostra de 713 atletas de 23 desportos dos EUA, de diferentes níveis competitivos, identificaram maiores competências psicológicas naqueles que alcançaram o sucesso desportivo.

Por se tratar de um questionário que tende à auto avaliação positiva das habilidades de coping existe a possibilidade de o atleta produzir respostas socialmente desejáveis aos itens. No entanto, esta limitação pode ser reduzida com a não identificação no indivíduo no questionário ${ }^{15}$.

Diante do exposto, fica claro que a construção do perfil psicológico do atleta para lidar com situações estressantes demanda tempo e acompanhamento. Portanto, é essencial que os técnicos, desde as categorias de base, além da preparação física, tática e técnica, possibilitem também um fortalecimento mental, observando as necessidades do atleta não só durante a fase de competição como na de preparação e, caso seja necessário, introduza um psicólogo na comissão técnica para a constante avaliação e extração do máximo desempenho. 


\section{CONCLUSÃO}

Atletas estão expostos a diversas situações causadoras de estresse, e a maneira com que cada um lida com ele pode influenciar seu desempenho final. No presente estudo, a comparação das estratégias de coping entre atletas de alto rendimento e praticantes de futebol feminino demonstrou que, o primeiro grupo obteve os melhores scores nas dimensões: desempenho sob pressão $(p=0,048)$, concentração $(p=0,020)$ e confiança/motivação $(p=0,009)$.
Compreender de modo mais eficiente como esses atletas podem atingir melhores escores em todas as dimensões é essencial para que o esporte evolua. No entanto, ainda são necessários mais estudos na área, com grupos mais específicos, para que se consiga adequar à demanda de cada um deles.

Todos os autores declararam não haver qualquer potencial conflito de interesses referente a este artigo.

CONTRIBUIÇÕES DOS AUTORES: Cada autor contribuiu individual e significativamente para o desenvolvimento do manuscrito. PJOC (0000-0001-8590-5172)* contribuiu com a concepção do problema e proposição do método. MRR (0000-0003-4597-4266)*, LMV (0000-0003-1023-8488)* e RPS (0000-0003-1178-304X)* realizaram a revisão de literatura. MRR e RPS realizaram a coleta de dados. MRR, LMV e PJOC realizaram ativamente a discussão do artigo. PJOC e LMV realizaram a revisão e aprovação final do trabalho. Todos os autores contribuíram com o conceito intelectual do estudo. *Número ORCID (Open Researcher and Contributor ID).

\section{REFERÊNCIAS}

1. Matos D, Cruz, J, Almeida L. Excelência no desporto: Para uma compreensão da arquitectura psicológica dos atletas de elite. Motricidade. 2011;7(4):27-41.

2. Laborde S, Dosseville F, Allen MS. Emotional intelligence in sport and exercise: A systematic review. Scand J Med Sci Sports. 2015 Jun 24. doi:10.1111/sms.12510. [Epub ahead of print].

3. Nicholls AR, Perry JL, Calmeiro L. Precompetitive achievement goals, stress appraisals, emotions, and coping among athletes. J Sport Exerc Psychol. 2014;36(5):433-45

4. Arruda AFS, Moreira A, Nunes JA, Viveiros L, De Rose Junior D, Aoki MS. Monitoramento do nível de estresse de atletas da seleção brasileira de basquetebol feminino durante a preparação para a Copa América 2009. Rev Bras Med Esporte. 2013;19(1):44-7.

5. Birrer D, Morgan G. Psychological skills training as a way to enhance an athlete's performance in highintensity sports. Scand J Med Sci Sports. 2010;20(Suppl 2):78-87.

6. Stefanello J. Situações de estresse no vôlei de praia de alto rendimento: um estudo de caso com uma dupla olímpica. Rev Port Cien Desp. 2007; 7(2):232-44.

7. Lazarus RS, Folkman S. Stress, appraisal, and coping. New York: Springer Publishing Company; 1984.

8. Costa ES, Leal IP. Estratégias de coping em estudantes do ensino superior. Aná Psicológica. 2006;24(2):189-99.

9. Sordes-Ader F, Fsian H, Esparbès S, Tap P. Stratégies de coping et désirable sociale. Aprendizagem e desenvolvimento. 1996;4(15/16):165-73.

10. Antoniazzi AS, Dell'Aglio DD, Bandeira DR. O conceito de coping: uma revisão teórica. Est Psicologia. 1998;3(2):273-94.

11. Ryan-Wenger NM. A taxonomy of children's coping strategies: a step toward theory development. Am J Orthopsychiatry. 1992;62(2):256-63.12.

12. Verardi CEL, Santos AB, Nagamine KK, Carvalho TD, Miyazaki MCD. Burnout and coping among football players: before and during the tournament. Rev Bras Med Esporte. 2014;20(4):272-5.

13. Hill AP, Appleton PR. The predictive ability of the frequency of perfectionistic cognitions, self-oriented perfectionism, and socially prescribed perfectionism in relation to symptoms of burnout in youth rugby players. J Sports Sci. 2011;29(7):695-703.
14. Franzini F. Futebol é "coisa para macho"?: Pequeno esboço para uma história das mulheres no país do futebol. Rev Bras Hist. 2005;25(50):315-28.

15. Coimbra DR. processo de validação do questionário "athletic coping skills inventory" para a língua portuguesa do Brasil [dissertação]. Juiz de Fora, MG: Universidade Federal de Juiz de Fora; 2011.

16. smith r., schutz r, smoll f, ptacekj. development and validation of a multidimensional measure of sport-specific psychological skills: the athletic coping skills inventory-28. Int J Sport Exerc Psychol. 1995;17(4):379-98.

17. Pires DA, Samulski DM, Costa VT. Indicadores de burnout e das estratégias de coping em atletas de judô e natação. Rev Bras Psicol Esporte. 2011;4(1):15-31.

18. Epping-Jordan JE, Compas BE, Osowiecki DM, Oppedisano G, Gerhardt C, Primo K, et al. Psychological adjustment in breast cancer: processes of emotional distress. Health Psychol. 1999;18(4):315-26.

19. Smith R, Christensen D. Psychological skills as predictors of performance and survival in professional baseball. J Sport Exerc Psychol. 1995;17:399-415.

20. Serpa S, Palmeira AL. ACSI-28-Estudo das experiências desportivas. Lisboa: Laboratório de Psicologia do Desporto, Faculdade de Motricidade Humana, Universidade Técnica de Lisboa; 1997.

21. Coimbra DR, Bara Filho M, Andrade A, Miranda R. Habilidades psicológicas de coping em atletas brasileiros. Motricidade. 2013;9(1):95-106.

22. Nascimento Junior JRA, Gaion PA, Nakashima FS, Vieira LF. Análise do estresse psicológico pré-competitivo e estratégias de coping de jovens atletas de futebol de campo. Rev Bras Ci Mov. 2010;18(4):45-53

23. Bim RH, Nascimento Junior JRA, Amorim AC, Vieira JLL, Vieira LF. Estratégias de coping e sintomas de burnout em atletas de futsal de alto rendimento. Rev Bras Ci Mov. 2014;22(3):69-75.

24. Pensgaard AM, Duda JL. Sydney 2000: The interplay between emotions coping and the performance of olimpic-level athletes. Sport Psychol. 2003;17:253-67.

25. Nicholls AR, Polman RC. Coping in sport: a systematic review. J Sports Sci. 2007;25(1):11-31.

26. Nicholls AR, Polman R, Levy AR, Taylor J, Cobley S. Stressors, coping, and coping effectiveness: gender, type of sport, and skill differences. J Sports Sci. 2007;25(13):1521-30.

27. Mahoney MJ, Avener M. Psychology of the elite athlete: an exploratory study. Cognit Ther Res 1977;1:135-41 